\title{
Intraventricular hemorrhage clot clearance rate as an outcome predictor in patients with aneurysmal subarachnoid hemorrhage: A retrospective study
}

Hae Gi Park

Department of Neurosurgery, Severance Stroke Center, Severance Hospital, Yonsei University College of Medicine, Seoul

\section{Sunghan Kim}

Department of Neurosurgery, Bucheon St. Mary's Hospital, College of Medicine, The Catholic University of Korea, Seoul

\section{Joonho Chung}

Department of Neurosurgery, Severance Stroke Center, Severance Hospital, Yonsei University College of Medicine, Seoul

\section{Chang Ki Jang}

Department of Neurosurgery, Yongin Severance Hospital, Yonsei University College of Medicine, Yongin

\section{Keun Young Park}

Department of Neurosurgery, Severance Stroke Center, Severance Hospital, Yonsei University College of Medicine, Seoul

Jae Whan Lee ( $\sim$ leejw@yuhs.ac)

Department of Neurosurgery, Yongin Severance Hospital, Yonsei University College of Medicine, Yongin

\section{Research Article}

Keywords: aneurysmal subarachnoid hemorrhage, cerebral intraventricular hemorrhage, patient outcomes assessment, clot clearance rate, modified Graeb score

Posted Date: August 12th, 2021

DOl: https://doi.org/10.21203/rs.3.rs-785820/v1

License: (c) (1) This work is licensed under a Creative Commons Attribution 4.0 International License. Read Full License

Version of Record: A version of this preprint was published at BMC Neurology on December 1st, 2021. See the published version at https://doi.org/10.1186/s12883-021-02505-0. 


\section{Abstract \\ Background}

The development of intraventricular hemorrhage (IVH) in aneurysmal subarachnoid hemorrhage (aSAH) is linked with higher mortality and poor neurological recovery. Previous studies have investigated the effect of the amount and distribution of the initial IVH on the prognosis of aSAH. However, no studies have assessed the relationship between the changes in IVH over time and the prognosis of aSAH. The aim of this study was to analyze the effect of the clearance rate of $\mathrm{IVH}$, which can be represented by the IVH clot clearance rate (CCR), on the outcomes of aSAH.

\section{Methods}

The IVH CCR was calculated based on the difference between the initial and follow-up modified Graeb scores (mGS), which were assessed by initial and 7-day follow-up brain computed tomography, respectively. Poor functional outcome was defined as a modified Rankin Scale score of 3-6. Univariate and multivariable analyses were performed to assess the relationships between IVH CCR and other risk factors and the prognosis of patients. Receiver operating characteristic curve analysis was performed to identify cut-off values of IVH CCR for predicting poor functional outcome.

\section{Results}

In total, 196 consecutive patients were diagnosed with aSAH between January 2014 and March 2018. According to the inclusion and exclusion criteria, 67 patients were finally included in the study. The univariate analysis revealed that a lower IVH CCR $(p<0.001)$, higher initial mGS $(p<0.001)$, older age $(p<$ $0.001)$, higher initial Hunt and Hess grade $(p<0.001)$, presence of delayed infarction $(p=0.03)$, and presence of shunt-dependent hydrocephalus $(p=0.004)$ were significantly related to poor functional outcome. The multivariable analysis revealed that IVH CCR (odds ratio [OR] 0.941; $p=0.029$ ), initial mGS (OR 1.632; $p=0.043$ ), age (OR 1.561; $p=0.007$ ), initial Hunt and Hess grade (OR 227.296; $p=0.030)$, and delayed infarction (OR 5310.632; $p=0.023$ ) were independent predictors of poor functional outcome. Optimal cut-off values of IVH CCR and mGS for poor outcome were $36.27 \%$, and 13.5 , respectively (all $p<$ $0.001)$.

\section{Conclusions}

The IVH CCR might have an important predictive value on poor functional outcome in patients with aSAH and IVH, along with initial mGS, age, initial Hunt and Hess grade, and delayed infarction.

\section{Background}


Approximately $30-70 \%$ of all cases of aneurysmal subarachnoid hemorrhage (aSAH) are accompanied by intraventricular hemorrhage (IVH), and the development of IVH in aSAH is linked with higher mortality and poor neurological recovery [1-3]. Previous studies have shown that IVH contributes to poor prognosis by causing various complications such as hydrocephalus [1, 3-7], intracranial hypertension [8], fever $[9,10]$ and ventriculitis $[11,12]$. Additionally, IVH can cause shunt-dependent hydrocephalus, which requires permanent cerebrospinal fluid (CSF) diversion $[7,13,14]$.

Meanwhile, previous studies have investigated the relationship between the distribution of IVH and prognosis of aSAH, going one step beyond the simple evaluation of aSAH prognosis based on the presence or absence of IVH. Various scoring systems have been suggested for determining the amount of IVH to be used as prognostic values, namely the Fischer grade [15], LeRoux score [16] and Graeb score [17]. In particular, Czorlich et al. demonstrated that the Graeb score seems to be superior to the Fisher and Le Roux scores in predicting mortality in patients with aSAH [18]. The modified Graeb score (mGS) was subsequently developed for more detailed scoring of IVH in patients with IVH [19]. mGS was found to be superior to the original Graeb score in terms of IVH evaluation in predicting the clinical course and outcome of patients with aSAH [20].

Prior studies have suggested that initial IVH volume or density can cause poor outcomes and fatality in patients with aSAH $[4,7,21]$. However, no studies have assessed the relationship between the changes in IVH over time and the prognosis of aSAH. Since previous studies have shown that the presence and amount of IVH affects the prognosis of aSAH, we hypothesized that the IVH clot clearance rate (CCR) will also affect the clinical outcome of aSAH with IVH. In this study, IVH CCR, which indicates the change in IVH with time, was calculated using the sequential change in the amount of $\mathrm{mGS}$. We aimed to evaluate the IVH CCR as an outcome predictor for patients with aSAH and IVH by retrospectively analyzing the functional outcome at 3-month follow-up. Furthermore, outcome predictors that affect the prognosis of aSAH with IVH, other than IVH CCR, were also investigated.

\section{Methods}

\section{Patient selection}

We retrospectively reviewed prospectively collected data regarding intracerebral aneurysms from a single institution's database. In total, 196 consecutive patients were diagnosed with aSAH between January 2014 and March 2018 (Fig. 1). The inclusion criteria of this study were as follows: (1) SAH was caused by the rupture of intracerebral aneurysm; (2) aSAH was accompanied by IVH at the time of diagnosis; (3) the ruptured aneurysm was treated either by microsurgery or by an endovascular method; (4) follow-up brain computed tomography (CT) was performed within 7 days to assess the IVH CCR and; (5) modified Rankins Scale (mRS) score at 3 months after discharge was identifiable via medical records. The exclusion criteria were as follows: (1) patients with any history of stroke that may affect the prognosis of aSAH other than IVH, such as a history of treatment for a ruptured or unruptured aneurysm, or a history of ischemic stroke; (2) patients presenting with any stroke-related lesion that may affect the prognosis of 
aSAH other than IVH, such as intracerebral hemorrhage, cerebral infarction, or vascular malformation; (3) patients who died within 7 days of admission, and; (4) patients with clinical deterioration due to rebleeding of the aneurysm. Intraventricular hemorrhage was confirmed in 80 of 173 patients, and 23 patients did not undergo follow-up CT within 7 days of aneurysmal rupture. After excluding 13 patients according to the exclusion criteria, 67 patients were finally included in the study.

Demographic, clinical, and radiographic data of the patients were collected based on electrical medical records, and data obtained from the aneurysm database were reviewed and compared for each group. The clinical condition on admission was graded according to the Hunt and Hess $(\mathrm{H}-\mathrm{H})$ grading system and radiologic condition on admission was assessed by SAH thickness on initial CT. The main outcome variable was the mRS score at 3-month follow-up, which was dichotomized between mRS scores of $0-2$ and 3-6. The included patients were divided into two groups according to the mRS score at 3-month follow up (good outcome group, mRS score of $0-2$ vs. poor outcome group, mRS score of 3-6). Delayed ischemic neurologic deficit (DIND), delayed infarction, and shunt-dependent hydrocephalus were investigated along with IVH CCR as prognostic factors for aSAH. Delayed ischemic neurologic deficit refers to symptomatic vasospasm that requires treatment identified by angiography, based on Kramer's criteria [1]. Delayed infarction was diagnosed if permanent cerebral infarction caused by DIND was confirmed by imaging. Shunt-dependent hydrocephalus was determined by clinical and radiologic signs of hydrocephalus which requires permanent CSF diversion within the follow-up period. This retrospective study was approved by the institutional review board of Severance hospital at Yonsei University College of Medicine (subject number: 4-2021-0828) and Bucheon St. Mary's hospital at the Catholic University of Korea (subject number: HC21RASI0072), and the requirement for informed consent was waived.

\section{Treatment strategies for aneurysmal subarachnoid hemorrhage}

All aSAH cases included in this study were treated according to the following protocol: (1) ruptured cerebral aneurysms were repaired within 48 hours by microsurgery or endovascular method; (2) the treatment plan was determined by multidisciplinary discussions among experienced neurosurgeons, neurointerventionists, and radiologists; (3) optimal medical treatment for preventing post-aSAH complications such as cerebral edema, vasospasm, and hydrocephalus were performed including close monitoring in intensive care units, administration of Nimodipine, and regular follow-up via transcranial doppler ultrasonography; (4) in case of acute hydrocephalus with clinical deterioration despite treatment, extraventricular drainage (EVD) was considered; (5) the EVD was maintained for up to two weeks, and if the EVD was intended to be maintained for more than two weeks, a change of EVD or permanent CSF diversion was considered contextually and; (6) the injection of intraventricular tissue plasminogen activator for the purpose of clot lysis was not considered.

\section{Assessment of the clot clearance rate of intraventricular hemorrhage}


The mGS was calculated by the method introduced by Morgan et al. [19]. The mGS incorporates IVH scores in relation to its anatomical extension. The maximum score of 32 indicates that every compartment is filled with blood and expanded. A score of 0 indicates no IVH. To evaluate the IVH CCR, initial and follow-up mGS was assessed by initial and 7-day follow-up brain CT, respectively. The IVH CCR was calculated as follows:

IVH CCR (\%): [(initial mGS - follow up mGS) / initial mGS] x 100

All mGS values were measured by two independent investigators. If the measurements of the two investigators differed considerably, another neuroradiologist reviewed the data and a final consensus was reached by the three investigators regarding the results.

\section{Statistical analysis}

A univariate analysis was performed to determine the association of poor outcome with factors and characteristics associated to aSAH with IVH. The Student's t-test was used for analyses of continuous variables and Pearson $\chi 2$ test was used for the analyses of categorical variables. Continuous variables were described as mean \pm standard deviation (SD) and categorical variables were summarized as frequencies and percentages (\%). A multivariate logistic regression analysis was performed for variables with an unadjusted effect, and $p$ values $<0.05$ in the univariate analysis was used to determine independent associations between poor outcome and characteristics of aSAH with IVH. The threshold value for the cut-off point of the continuous variable among the outcome predictors for aSAH with IVH was determined using the receiver operating characteristics (ROC) analysis, and the diagnostic performance of the each variable was assessed. Pearson's rank correlation coefficient was used to extrapolate the relationships between the continuous variable among the outcome predictors and IVH CCR. All $p$ values $<0.05$ were considered statistically significant. All statistical analyses were performed using SPSS Statistics 23.0 (IBM Corp., Amonk, NY, USA).

\section{Results}

\section{Outcome predictors for aneurysmal subarachnoid hemorrhage with intraventricular hemorrhage}

Among the 67 patients, poor outcome was observed in 29 (43.3\%) patients. The univariate analysis revealed that old age $(p<0.001)$, higher initial $\mathrm{H}-\mathrm{H}$ grade $(p<0.001)$, presence of delayed infarction $(p=0.03)$, and presence of shunt-dependent hydrocephalus $(p=0.004)$ were significantly associated with poor functional outcome. However, sex, SAH thickness, location of aneurysm, and DIND were not significantly related to poor functional outcome. The initial $\mathrm{mGS}$ of the poor outcome group was higher than that of the good outcome group ( $16.8 \pm 5.7$ vs. $10.6 \pm 5.4, p<0.001)$. The IVH CCR in the poor outcome group was lower than that in the good outcome group ( $28.3 \pm 24.0$ vs. $67.4 \pm 29.1, p<0.001)$. The multivariable analysis revealed that old age (odds ratio [OR], $1.561 ; 95 \%$ confidence interval [Cl], 1.126- 
2.162; $\mathrm{p}=0.007)$, higher initial $\mathrm{H}-\mathrm{H}$ grade $(\mathrm{OR}, 227.296 ; 95 \% \mathrm{Cl} 1.715-30119.199 ; \mathrm{p}=0.030)$, and presence of delayed infarct (OR, 5310.632; 95\% $\mathrm{Cl} 3.209-8788298.374 ; \mathrm{p}=0.023)$ were independent predictors of poor outcome. Additionally, a higher initial mGS (OR, 1.632; 95\% Cl, 1.015-2.622; $\mathrm{p}=0.043)$ and low rate of IVH CCR $(O R, 0.941 ; 95 \% \mathrm{Cl}, 0.890-0.994 ; \mathrm{p}=0.029)$ were independent predictors of unfavorable functional outcome. These results are summarized in Table 1.

\section{Associations of outcome predictors and functional outcomes of aneurysmal subarachnoid hemorrhage with intraventricular hemorrhage}

The ROC curve for the cut-off value of the IVH CCR showed an area under the curve (AUC) of 0.838 (Fig. 2a). The cut-off value of the IVH CCR was determined to be $36.27 \%$, for which the sensitivity and specificity were $84.2 \%$ and $75.9 \%$ respectively. The ROC curve for the initial mGS for the prediction of poor outcome in aSAH with IVH showed an AUC of 0.787 (Fig. 2b). The cut-off value of the initial mGS was determined to be 13.5 , for which the sensitivity and specificity were $75.9 \%$ and $68.4 \%$ respectively. Pearson's rank correlation coefficient was used to extrapolate the relationships between initial mGS (Fig. 3a), age (Fig. 3b), and IVH CCR. The correlation test revealed a negative correlation for both initial mGS $(r=-0.422, p<0.001)$ and age $(r=-0.336, p<0.001)$ with IVH CCR.

\section{Discussion}

In this observational cohort of 67 patients with aSAH and IVH, it was found that a low rate of IVH CCR independently predicted unfavorable functional outcome in patients with aSAH and IVH along with old age, higher initial $\mathrm{H}-\mathrm{H}$ grade, presence of delayed infarction, and higher initial mGS. Furthermore, initial mGS was found to be negatively correlated with IVH CCR. These results suggest that not only initial IVH volume or density but also IVH CCR is related to the functional outcome in aSAH with IVH.

Previous studies have reported the impact of initial IVH burden on early complications and fatality in cases of aSAH. Czorlich et al.[18] retrospectively analyzed aSAH in 206 consecutive patients and discussed a significant association between initial IVH severity and case fatality rate. Moreover, Darkwah Oppong et al.[4] reported the result of a retrospective study of aSAH with IVH in 487 patients that severity of IVH was related to acute hydrocephalus and poor outcome at 5-month follow-up, and that the outcome was closely related to early complications of aSAH such as cerebral infarction, early mortality, and primary craniectomy. Eagles et al.[22] observed that delayed cerebral ischemia in aSAH can be predicted by initial IVH volume calculated using mGS. As IVH adversely affects the prognosis of aSAH in various ways, it can be hypothesized that if IVH clearance is delayed, these adverse effects will be maintained for a long time, resulting in a poor prognosis. This hypothesis is corroborated by the current findings that a low rate of IVH CCR is an independent prognostic factor of poor functional outcome in aSAH with IVH.

Hemorrhaging that extends to the ventricular system damages the brain via several possible mechanisms. The severity of hemorrhaging has a direct mass effect on the adjacent neural tissue, which 
can cause neural damage and cerebral edema [23]. Moreover, hemorrhage in the ventricular system blocks the circulatory balance of the CSF, and it can negatively affect the regulation of intracranial pressure and cerebral perfusion [24]. IVH also results in an increased inflammatory reaction in the CSF [25]. Moreover, the greater is the initial IVH burden, the greater is the CSF inflammatory reaction. Therefore, a slower IVH CCR might result in additional exposure with respect to the CSF inflammatory reaction. This might be the one of reasons why the IVH CCR in the early hemorrhage period is related to poor functional outcome, as shown by our results.

There were several limitations to this study. First, it was designed retrospectively and included a small number of patients from a single center. Second, even though two observers assessed the lesions independently, bias could have affected the results. Third, the efficacy of aneurysm treatments performed, such as clips and coils, is expected to affect the patient's prognosis; however, this was not considered in this study. Finally, the effect of CSF drainage on IVH CCR and patient outcomes was not evaluated. Therefore, further prospective studies with well-adjusted confounding variables are needed to better understand the relationship between IVH CCR and functional outcomes of aSAH with IVH.

\section{Conclusions}

The results of this study suggest that the IVH CCR might have an important predictive value on poor functional outcome in patients with aSAH and IVH, along with age, $\mathrm{H}-\mathrm{H}$ grade, delayed infarct, and initial mGS. Additional studies with larger sample sizes are needed to provide more evidence in this regard.

\section{Abbreviations}

aSAH: aneurysmal subarachnoid hemorrhage; AUC: area under the curve; CCR: clot clearance rate; $\mathrm{Cl}$ : confidence interval; CSF: cerebrospinal fluid; CT: computed tomography; DIND: Delayed ischemic neurologic deficit; EVD: extraventricular drainage; $\mathrm{H}-\mathrm{H}$ : Hunt and Hess; IVH: intraventricular hemorrhage; mGS: modified Graeb score; mRS: modified Rankins Scale; OR: odds ratio; ROC: receiver operating characteristics; SD: standard deviation

\section{Declarations}

\section{Ethics approval and consent to participate}

This study was approved by the Instituitional Research Ethics Committee of Severance hospital in Yonsei University College of Medicine (subject number: 4-2021-0828) and Bucheon St. Mary's hospital in the Catholic University of Korea (subject number: HC21RASI0072). All procedures performed in the studies involving human participants were in accordance with the ethical standards of our Institutional Review Board with the 1964 Helsinki Declaration and its later amendments or comparable ethical standards. In this retrospective study, the requirement for informed consent was waived. Patient data was de-identified data upon data collection. 


\section{Consent for publication}

Not applicable.

\section{Availability of data and materials}

The relevant anonymized patient level data are available upon reasonable request from the authors.

\section{Competing interests}

The authors declare that they have no conflict of interest.

\section{Funding}

No funding was received for this research.

\section{Authors' contributions}

HGP and SK contributed to the concept of the study, interpretation of data, statistical analysis, drafting of the manuscript, and revision of the manuscript. CKJ contributed to the acquisition of data, analysis of data, and revision of the manuscript. KYP and $\mathrm{JC}$ contributed to the interpretation of data and revision of the manuscript. JWL contributed to the concept of the study, revising and editing the manuscript, and supervising the whole process of the study. All authors have read and approved the final version of the manuscript.

\section{Acknowledgements}

We would like to thank Editage (www.editage.co.kr) for English language editing.

\section{References}

1. Kramer AH, Mikolaenko I, Deis N, Dumont AS, Kassell NF, Bleck TP, et al. Intraventricular hemorrhage volume predicts poor outcomes but not delayed ischemic neurological deficits among patients with ruptured cerebral aneurysms. Neurosurgery. 2010;67:1044-52; discussion 52 - 3. 
2. Mayfrank L, Hutter BO, Kohorst Y, Kreitschmann-Andermahr I, Rohde V, Thron A, et al. Influence of intraventricular hemorrhage on outcome after rupture of intracranial aneurysm. Neurosurg Rev. 2001;24:185-91.

3. Rosen DS, Macdonald RL, Huo D, Goldenberg FD, Novakovic RL, Frank JI, et al. Intraventricular hemorrhage from ruptured aneurysm: clinical characteristics, complications, and outcomes in a large, prospective, multicenter study population. J Neurosurg. 2007;107:261-5.

4. Darkwah Oppong M, Gembruch O, Herten A, Frantsev R, Chihi M, Dammann P, et al. Intraventricular Hemorrhage Caused by Subarachnoid Hemorrhage: Does the Severity Matter? World Neurosurg. 2018;111:e693-e702.

5. Lee VH, Ouyang B, John S, Conners JJ, Garg R, Bleck TP, et al. Risk stratification for the in-hospital mortality in subarachnoid hemorrhage: the HAIR score. Neurocrit Care. 2014;21:14-9.

6. Nguyen HS, Li L, Patel M, Kurpad S, Mueller W. Radiodensity of intraventricular hemorrhage associated with aneurysmal subarachnoid hemorrhage may be a negative predictor of outcome. $J$ Neurosurg. 2018;128:1032-6.

7. Wilson TJ, Stetler WR, Jr., Davis MC, Giles DA, Khan A, Chaudhary N, et al. Intraventricular hemorrhage is associated with early hydrocephalus, symptomatic vasospasm, and poor outcome in aneurysmal subarachnoid hemorrhage. J Neurol Surg A Cent Eur Neurosurg. 2015;76:126-32.

8. Heuer GG, Smith MJ, Elliott JP, Winn HR, LeRoux PD. Relationship between intracranial pressure and other clinical variables in patients with aneurysmal subarachnoid hemorrhage. J Neurosurg. 2004;101:408-16.

9. Fernandez A, Schmidt JM, Claassen J, Pavlicova M, Huddleston D, Kreiter KT, et al. Fever after subarachnoid hemorrhage: risk factors and impact on outcome. Neurology. 2007;68:1013-9.

10. Dorhout Mees SM, Luitse MJ, van den Bergh WM, Rinkel GJ. Fever after aneurysmal subarachnoid hemorrhage: relation with extent of hydrocephalus and amount of extravasated blood. Stroke. 2008;39:2141-3.

11. Bota DP, Lefranc F, Vilallobos HR, Brimioulle S, Vincent JL. Ventriculostomy-related infections in critically ill patients: a 6-year experience. J Neurosurg. 2005;103:468-72.

12. Frontera JA, Fernandez A, Schmidt JM, Claassen J, Wartenberg KE, Badjatia N, et al. Impact of nosocomial infectious complications after subarachnoid hemorrhage. Neurosurgery. 2008;62:80-7; discussion 7.

13. Della Pepa GM, Scerrati A, Albanese A, Marchese E, Maira G, Sabatino G. Protective effect of external ventricular drainage on cerebral vasospasm. A retrospective study on aneurysmal SAH treated endovascularly. Clin Neurol Neurosurg. 2014;124:97-101.

14. Yang TC, Chang CH, Liu YT, Chen YL, Tu PH, Chen HC. Predictors of shunt-dependent chronic hydrocephalus after aneurysmal subarachnoid haemorrhage. Eur Neurol. 2013;69:296-303.

15. Fisher CM, Kistler JP, Davis JM. Relation of cerebral vasospasm to subarachnoid hemorrhage visualized by computerized tomographic scanning. Neurosurgery. 1980;6:1-9. 
16. LeRoux PD, Haglund MM, Newell DW, Grady MS, Winn HR. Intraventricular hemorrhage in blunt head trauma: an analysis of 43 cases. Neurosurgery. 1992;31:678 - 84; discussion $84-5$.

17. Graeb DA, Robertson WD, Lapointe JS, Nugent RA, Harrison PB. Computed tomographic diagnosis of intraventricular hemorrhage. Etiology and prognosis. Radiology. 1982;143:91-6.

18. Czorlich P, Ricklefs F, Reitz M, Vettorazzi E, Abboud T, Regelsberger J, et al. Impact of intraventricular hemorrhage measured by Graeb and LeRoux score on case fatality risk and chronic hydrocephalus in aneurysmal subarachnoid hemorrhage. Acta Neurochir (Wien). 2015;157:409-15.

19. Morgan TC, Dawson J, Spengler D, Lees KR, Aldrich C, Mishra NK, et al. The modified graeb score: an enhanced tool for intraventricular hemorrhage measurement and prediction of functional outcome. Stroke. 2013;44:635-41.

20. Czorlich P, Mende KC, Vettorazzi E, Regelsberger J, Westphal M, Schmidt NO. Validation of the modified Graeb score in aneurysmal subarachnoid hemorrhage. Acta Neurochir (Wien). 2015;157:1867-72; discussion 72.

21. Inagawa T, Shibukawa M, Hidaka T. A Comparison of computed tomography-based scales with and without consideration of the presence or absence of intraventricular hemorrhage in patients with aneurysmal subarachnoid hemorrhage. World Neurosurg. 2018;114:e926-e37.

22. Eagles ME, Jaja BNR, Macdonald RL. Incorporating a modified graeb score to the modified fisher scale for improved risk prediction of delayed cerebral ischemia following aneurysmal subarachnoid hemorrhage. Neurosurgery. 2018;82:299-305.

23. Wagner I, Volbers B, Hilz MJ, Schwab S, Doerfler A, Staykov D. Radiopacity of intracerebral hemorrhage correlates with perihemorrhagic edema. Eur J Neurol. 2012;19:525-8.

24. Mohr G, Ferguson G, Khan M, Malloy D, Watts R, Benoit B, et al. Intraventricular hemorrhage from ruptured aneurysm. Retrospective analysis of 91 cases. J Neurosurg. 1983;58:482-7.

25. Fam MD, Zeineddine HA, Eliyas JK, Stadnik A, Jesselson M, McBee N, et al. CSF inflammatory response after intraventricular hemorrhage. Neurology. 2017;89:1553-60.

\section{Tables}

Table 1. Univariate and multivariable analysis of risk factors for poor functional outcomes after aneurysmal subarachnoid hemorrhage with intraventricular hemorrhage 


\begin{tabular}{|c|c|c|c|c|c|}
\hline & & & $\begin{array}{l}\text { Univariate } \\
\text { analysis }\end{array}$ & $\begin{array}{c}\text { Multivariable } \\
\text { analysis }\end{array}$ & \\
\hline Parameter & $\begin{array}{c}\text { Good } \\
\text { outcome } \\
(n=38)\end{array}$ & $\begin{array}{c}\text { Poor } \\
\text { outcome } \\
(n=29)\end{array}$ & P-value & OR $(95 \% \mathrm{CI})$ & $\begin{array}{c}\mathrm{P}- \\
\text { value }\end{array}$ \\
\hline Age (years) & $55.9 \pm 11.3$ & $74.5 \pm 8.9$ & $<0.001$ & $\begin{array}{c}1.561(1.126- \\
2.162)\end{array}$ & 0.007 \\
\hline Female & $33(86.8)$ & $22(75.9)$ & 0.200 & & \\
\hline Initial $\mathrm{H}-\mathrm{H}$ grade & & & $<0.001$ & $\begin{array}{c}227.296(1.715- \\
30119.199)\end{array}$ & 0.030 \\
\hline $1-3$ & $33(86.8)$ & $6(20.7)$ & & & \\
\hline $4-5$ & $5(13.2)$ & $23(79.3)$ & & & \\
\hline SAH thickness & & & 0.173 & & \\
\hline Thin $(<1 \mathrm{~mm})$ & $16(42.1)$ & $12(41.4)$ & & & \\
\hline Thick (>1mm) & $22(57.9)$ & $17(58.6)$ & & & \\
\hline $\begin{array}{l}\text { Location } \quad \text { of } \\
\text { aneurysm }\end{array}$ & & & 0.200 & & \\
\hline $\begin{array}{l}\text { Anterior } \\
\text { circulation }\end{array}$ & $33(86.8)$ & $22(75.9)$ & & & \\
\hline $\begin{array}{l}\text { Posterior } \\
\text { circulation }\end{array}$ & $5(13.2)$ & $7(24.1)$ & & & \\
\hline DIND & $3(7.9)$ & $3(10.3)$ & 0.526 & & \\
\hline Delayed infarction & $2(5.3)$ & $7(24.1)$ & 0.030 & $\begin{array}{c}5310.632(3.209- \\
8788298.374)\end{array}$ & 0.023 \\
\hline $\begin{array}{l}\text { Shunt-dependent } \\
\text { hydrocephalus }\end{array}$ & $6(15.8)$ & $14(48.3)$ & 0.004 & $0.025(0-4.957)$ & 0.171 \\
\hline Initial mGS & $10.6 \pm 5.4$ & $16.8 \pm 5.7$ & $<0.001$ & $\begin{array}{c}1.632(1.015- \\
2.622)\end{array}$ & 0.043 \\
\hline IVH CCR (\%) & $67.4 \pm 29.1$ & $28.3 \pm 24.0$ & $<0.001$ & $\begin{array}{c}0.941(0.890- \\
0.994)\end{array}$ & 0.029 \\
\hline
\end{tabular}

Values are presented as mean \pm standard deviation or number (\%). 
$O R$ odds ratio; $C I$ confidence interval; $H$ - $H$ Hunt \& Hess; $S A H$ subarachnoid hemorrhage; $C H C$ chronic hydrocephalus; DIND delayed ischemic neurologic deficiet; mGS modified Graeb score; $I V H$ intraventricular hemorrhage; $C C R$ intraventricular clot clearance rate

\section{Figures}

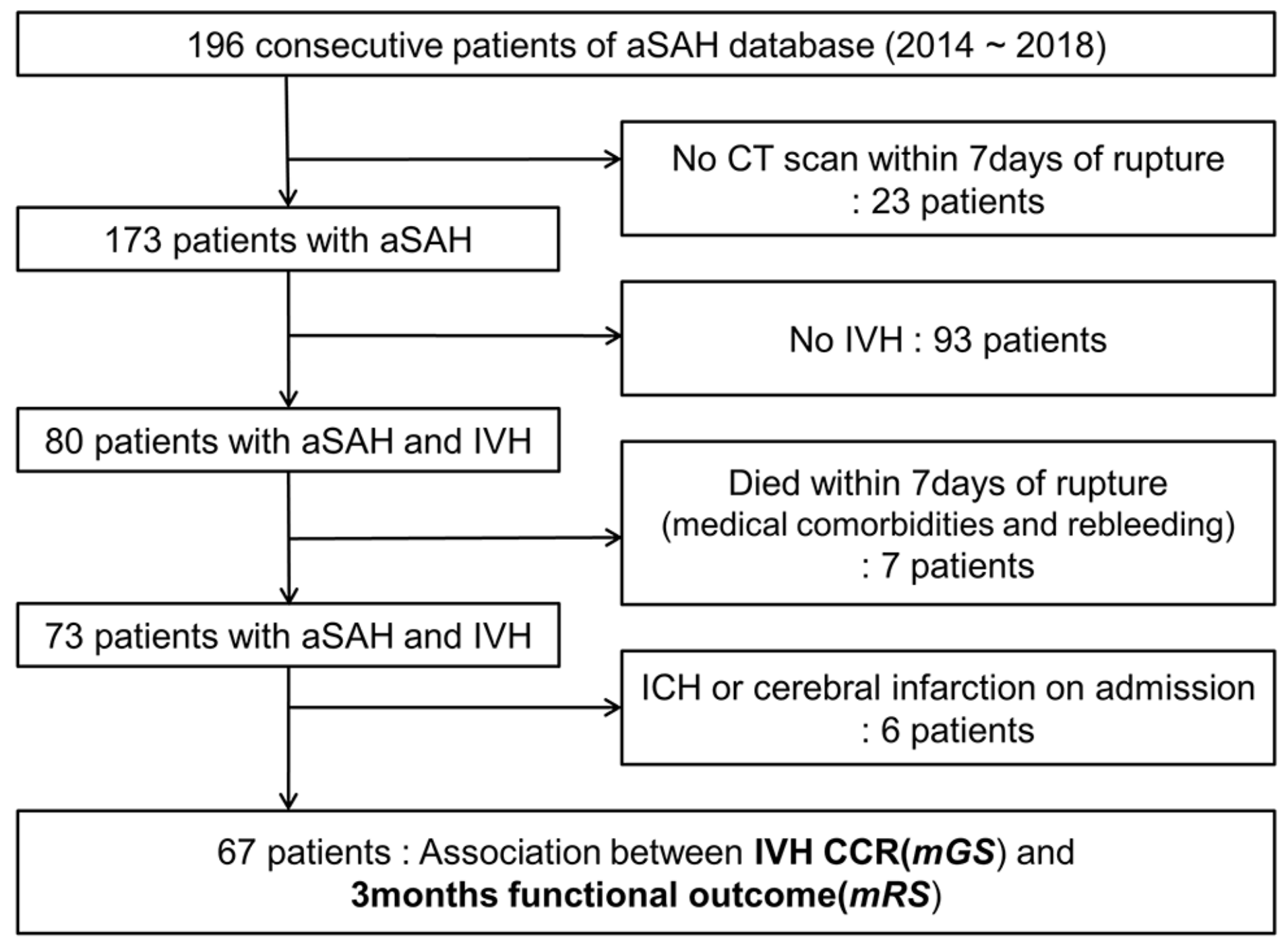

Figure 1

Description of the patient cohort used in this study. IVH intraventricular hemorrhage; CCR clot clearance rate; aSAH aneurysmal subarachnoid hemorrhage; CT computed tomography; ICH intracerebral hemorrhage; mGS modified Graeb Scale; mRS modified Rankins Scale 

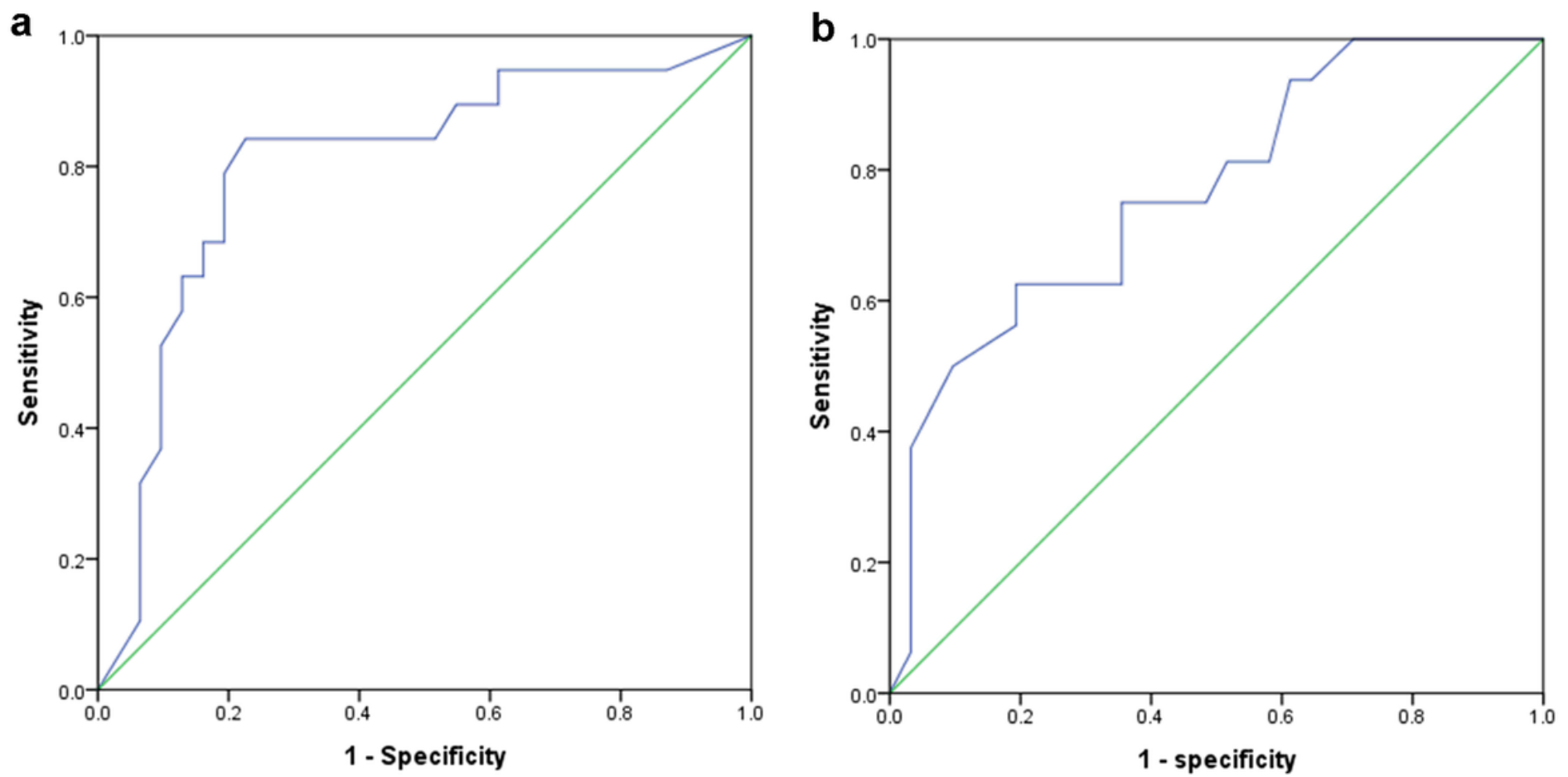

Figure 2

The ROC curve of the IVH CCR and the initial mGS for the outcome prediction. (a) The cut-off value of the IVH CCR was $36.27 \%$ (AUC 0.838, P< 0.001). (b) The cut-off value of the initial mGS was 13.5 (AUC 0.787 , $\mathrm{P}<0.001)$. ROC receiver operating characteristic; IVH intraventricular hemorrhage; CCR intraventricular clot clearance rate; mGS modified Graeb score; AUC area under the curve
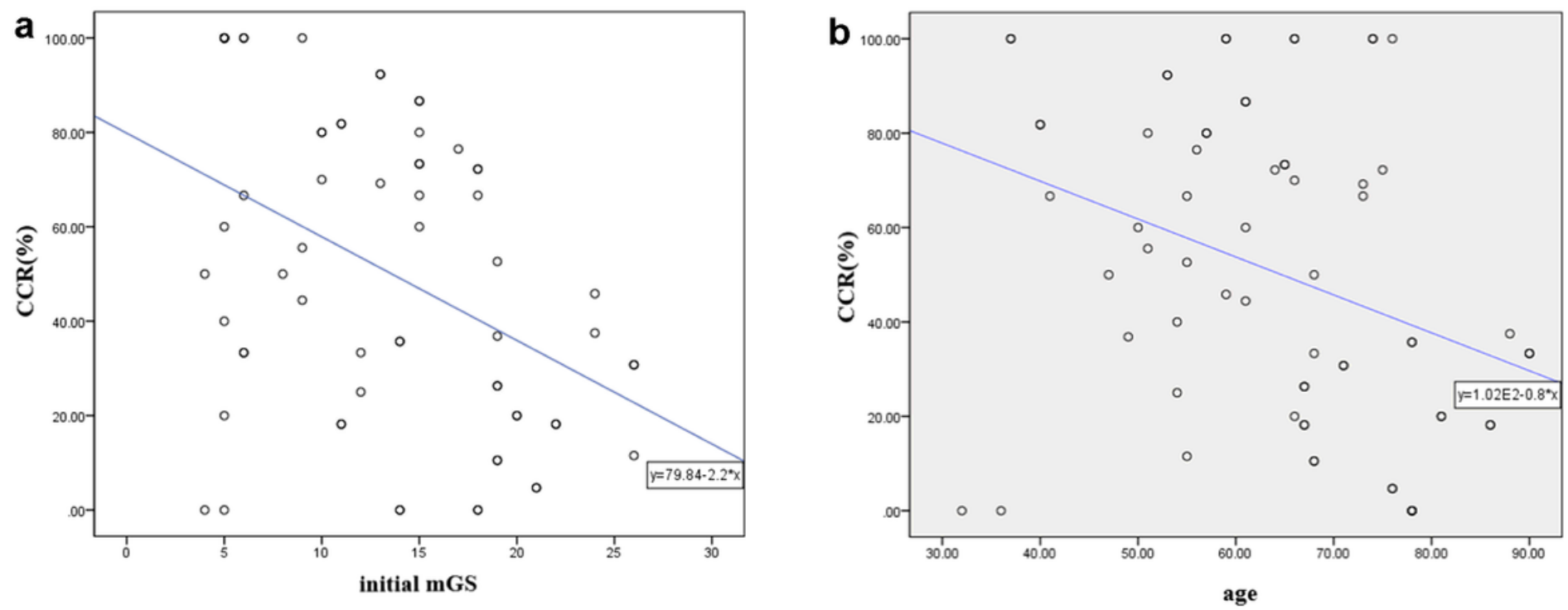

Figure 3

Correlation of (a) initial mGS, (b) age, and IVH CCR. A negative correlation was noted for both initial mGS $(r=-0.422, p<0.001)$ and age $(r=-0.336, p<0.001)$ with IVH CCR. mGS modified Graeb score; IVH 
intraventricular hemorrhage; CCR intraventricular clot clearance rate 